\title{
Foot stress fracture due to pregnancy-induced osteoporosis
}

\begin{abstract}
Pregnancy-induced osteoporosis is a rare, although wellknown complication of pregnancy. Fractures may result that can cause invalidating pain. We describe a case of a patient who presented on the 39th week with persistent right foot pain and was diagnosed with a stress fracture of the second metatarsal. After complete work-up and elimination of all other pathologies, the fracture was considered to be due to pregnancy-related osteoporosis. The Patient was managed conservatively with reduced weightbearing and protective footwear. Stress fractures during pregnancy related to osteoporosis though rare, should be easily recognised in order to accelerate recovery and prevent complications.
\end{abstract}

Keywords: stress fracture, pregnancy-induced osteoporosis, X-ray
Volume 2 Issue 2 - 2015

\author{
Helge Burmeister,' Maria Zormpa,' George \\ Lazarou $^{3}$ \\ 'Department of Obstetrics and Gynecology, Hospital of \\ Langenthal, Switzerland \\ ${ }^{2}$ Tell-Klinik AG, Switzerland \\ ${ }^{3}$ Director of Urogynecology \& Reconstructive Pelvic Surgery, \\ Winthrop University Hospital, USA
}

Correspondence: Maria Zormpa, Hospital of Langenthal, St. Urbanstrasse 67, 490 I Langenthal, Switzerland, Tel +4I-062-9|83893, Fax 062-918-43-90, Email mariazormpa7@gmail.com

\section{Introduction}

Pregnancy-induced osteoporosis is a recognised entity with an unknown pathophysiology that is a diagnosis of exclusion. Fractures occur rarely when predisporing factors are absent. The presence of predisposing factors leads to stress fractures. Causes of stress fractures are excessive exercise, glucocorticoid intake, alcohol abuse, smoking, renal disease, endocrine and hormonal imbalances. ${ }^{1}$ In addition, women with extreme weight gain, increase of sport activities during pregnancy, macrosomic infants or assisted vaginal delivery run a higher risk for fractures. ${ }^{2-5}$ We present a 34-year-old patient who had none of the previously mentioned risk factors and developed a foot stress fracture in the third trimester. We attribute the fracture to pregnancy-induced osteoporosis.

\section{Case presentation}

A 34-year-old Caucasian trigravid patient in the $39^{\text {th }}$ week of pregnancy with a history of two c-sections was admitted for a planned c-section. She originally experienced bilateral foot pain for five weeks and then a sudden stabbing sensation and swelling in the right foot proximally three days before the admission (Figure 1). The patient mentioned that she suddenly had bilateral foot pain without having any kind of accident. The pain was exacerbated with weight-bearing and the patient had to use crutches to alleviate the pain and rest as much as possible at home. Additionally, she used paracetamol for pain relief. On admission, the patient had a total weight gain of 7kilos in the pregnancy and her BMI was $26.1 \mathrm{~kg} / \mathrm{m}^{2}$. The estimated fetal weight on ultrasound was about $2900 \mathrm{gr}$. She did not use any alcohol, tabacco or illegal drugs and did regular exercise for one hour three times a week before getting pregnant. The patient had been using prenatal vitamins and had balanced diet containg 3-4 snacks of milk products per day throughout the pregnancy. She walked for half an hour twice a week since the first trimester did not do any other kind of workout. She had no history of a prior fracture or over use, malignancy, endocrine, gastrointestinal (Crohn's or celiac disease), renal or autoimmune disorder. There was no family history of bone disease. She denied any history of trauma or injuries during exercising.

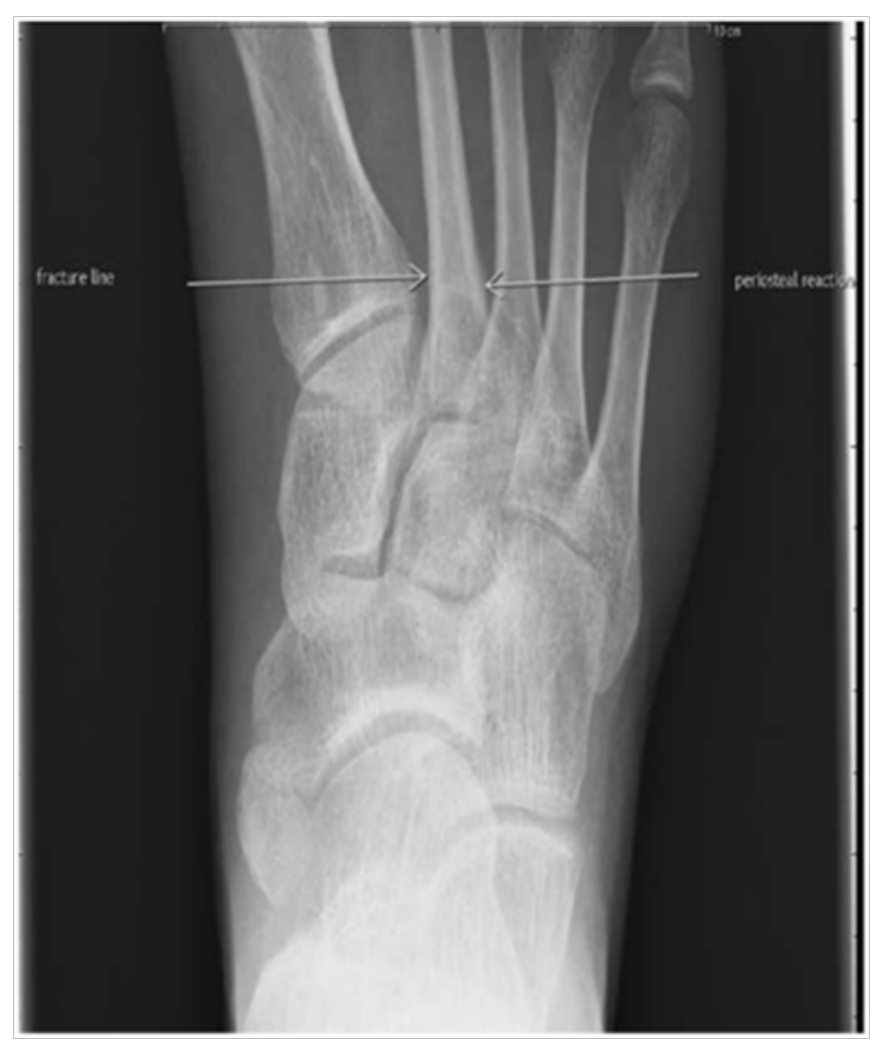

Figure I Anteroposterior view of the right foot. Fracture line in the base of second metatarsal and periosteal reaction.

\section{Discussion}

Pregnancy-associated osteoporosis has a prevalence of $0.4 / 100.000 .{ }^{6}$ Studies shedding light on the pathophysiology have been conducted since the 1950s and support that fractures occur in women with already decreased bone strength. ${ }^{7}$ Genetics are a contributing factor, such as deficiency of calcitonin or its receptor and elevated 
levels of parathyroid hormone-related protein enhance osteoclast activation. ${ }^{8,9}$ Another possible mechanism is vascular injury leading to ischemia and marrow edema. ${ }^{10}$ The diagnosis can be suspected in case of severe foot pain during pregnancy. Other suspicious areas for fractures are the hips, pelvis and ribs. ${ }^{11}$ Full laboratory workup should be performed to exclude renal and thyroid disorders, HIV, malnutrition, malignancy and autoimmune diseases and drug-related osteoporosis should also be eliminated. ${ }^{12}$ Unfractionated heparin for prevention of thromboembolic disease in case of thrombophilia is also associated with higher risk of osteoporosis. ${ }^{13} \mathrm{X}$-ray is the first radiologic investigation to take place and DXA scan of the lumbar spine and hip it the modality of choice. ${ }^{14}$ Drugs suggested for the management of pregnancy-related osteoporosis include calcium and vitamin $\mathrm{D}$, calcitonin, teriparatide and strontium ranelate., ${ }^{7,15}$ It has also been supported that biphosphonates lead to a significant increase in bone density. ${ }^{17,18}$ Nevertheless, as the condition is self-limited and there is a very limited number of data, no intervention is suggested unless the patient suffers from vertebral fractures at a young age. ${ }^{19,20}$ The risk of recurrence in subsequent pregnancies is low and the patients recover usually within a year. ${ }^{21,22}$ However, timely diagnosis of stress fractures due to pregnancy-induced osteoporosis is essential to alleviate the patient from severe pain and facilitate fast recovery.

\section{Acknowledgments}

None.

\section{Conflicts of interest}

The authors declare there is no conflict of interests.

\section{References}

1. Bennell K, Matheson G, Meeuwisse W, et al. Risk factors for stress factors. Sports Med. 1999;28(2):91-122.

2. Mikawa Y, Watanabe R, Yamano Y, et al. Stress fractures of the body of the pubis in a pregnant woman: case report. Arch Orthop Trauma Surg. 1998;107(3):193-194.

3. Moran JJ. Stress fractures in pregnancy. Am J Obstet Gynecol. 1988;158(6 Pt 1):1274-1277.

4. Breuil V, Brocq O, Euller-Ziegler L, et al. Insufficiency fracture of the sacrum revealing a pregnancy-associated osteoporosis: a first case report. Ann Rheum Dis. 1997;56(4):278-279.

5. Bushbaum HJ. Healing of experimental fractures during pregnancy. Obstet Gynecol. 1977;35:613-615.

6. Pishnamaz M, Sellei R, Pfeifer R, et al. Low back pain during pregnancy caused by a sacral stress fracture: a case report. J Med Case Rep. 2012;6:98.
7. Kovacs CS. Calcium and bone metabolism disorders during pregnancy and lactation. Endocrinol Metab Clin North Am. 2011;40(4):795-826.

8. Dunne F, Walters B, Marshall T, et al. Pregnancy-associated osteoporosis. Clin Endocrinol (Oxf). 1993;39(4):487-490.

9. Anai T, Tomiyasu T, Arima K, et al. Pregnancy-associated osteoporosis with elevated levels of parathyroid-hormone related protein: a report of two cases. J Obstet Gynaecol Res. 1999;25(1):63-67.

10. Rishi V, Wahl P, Mahajan A. Transient osteoporosis of hip (migratory). JK Science. 2008;10(4):194-196.

11. Ozturk G, Kulcu DG, Aydog E. Intrapartum sacral stress fracture due to pregnyncy-related osteoporosis: a case report. Arch Osteoporos. 2013;8(1-2):139.

12. European Society of Endocrinology. Clinical and translational endocrinology from around the world. 2nd ed. 2010.

13. Barbour LA, Kick SD, Steiner JF, et al. A prospective study of heparininduced osteoporosis in pregnancy using bone density. Am J Obstet Gynecol. 1994;170(3):862-869.

14. Khan AA, Hanley DA, Bilezikian JP, et al. Standards for performing DXA in individual with secondary causes of osteoporosis. J Clin Densitom. 2006;9(1):47-57.

15. Hellmeyer L, Boekhoff J, Hadji P. Treatment with teriparatide in a patient with pregnancy-associated osteoporosis. Gynecol Endocrinol. 2010;26(10):725-728.

16. Tanriover MD, Oz SG, Sozen T, et al. Pregnancy and lactation-associated osteoporosis with severe vertebral deformities: can strontium ranelate be a new alternative for treatment. Spine J. 2009;9(4):e20-e24.

17. Shenker NG, Shaikh MF, Jawad AS. Transient osteoporosis associated with pregnancy: use of biphosphonate in treating a lactating mother. BMJ. 2010.

18. O'Sullivan SM1, Grey AB, Singh R, et al. Biphosphonates in pregnancy and lactation-associated osteoporosis. Osteoporosis Int. 2006;17(7):1008-1012.

19. Davey MR, De Villiers T, Lipschitz S, et al. Pregnancy- and lactationassociated osteoporosis. JEMDSA. 2012;17(3):149-153.

20. Smith R, Philips AJ. Osteoporosis during pregnancy and its management Scand J Rheumatol Suppl. 1998;107:66-67.

21. Mahadevan S, Kumaravel V, Bharath R. Calcium and bone disorders in pregnancy. Indian J Endocrinol Metab. 2012;16(3):358-363.

22. Ma FY, Falkenberg M. Case reports: transient osteoporosis of the hip: an atypical case. Clin Orthop Relat Res. 2006;445:245-249. 